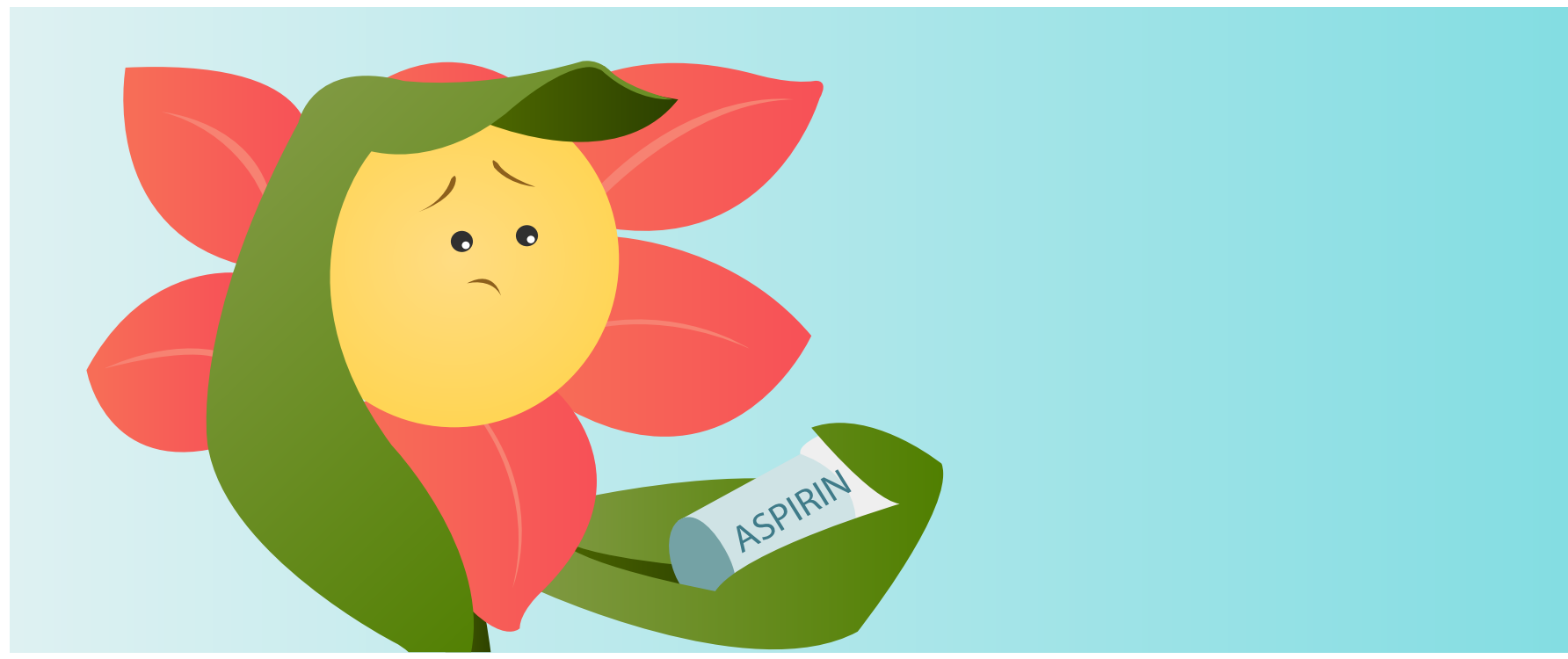

\title{
HOW DOES ASPIRIN WORK IN PLANTS AND
}

\section{HUMANS?}

\section{Daniel F. Klessig*}

Boyce Thompson Institute, Cornell University, Ithaca, NY, USA

REVIEWED BY:

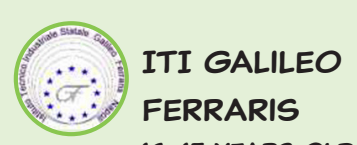

14-15 YEARS OLD
Millions of people rely on aspirin to treat their headaches, fevers, and other ailments. But most people do not know that the active ingredient in aspirin was first discovered in plants. In humans, the body converts aspirin into a substance called salicylic acid (SA). Plants make SA. They use it to help defend themselves against infection. To discover how SA works in plants, we used powerful methods to identify over two dozen proteins that bind to SA. SA binding changes the activities of these proteins. SA and related compounds also bind to multiple proteins in humans. We discovered several new human proteins that can bind SA. These proteins are associated with very common, devastating human diseases. In addition, we identified several new versions of SA that bind to these proteins more strongly than SA does. As a result, these versions of SA inhibit the disease-associated activities of these proteins better than SA. This gives hope that better aspirin-like drugs can be made.

\section{WHAT IS ASPIRIN?}

Aspirin is the most used medicine worldwide. About 80 million pounds of aspirin are produced and 100 billion tablets consumed each year. Aspirin reduces 
INFLAMMATION

Is part of the complex biological response of body tissues to harmful stimuli, such as pathogens, damaged cells, or irritants. It is a protective response involving immune cells.

\section{FIGURE}

Chemical structures of salicylic acid (SA) and its man-made and natural relatives.

The central part of the SA compound is a phenyl ring composed of six carbon (C) atoms at positions 1 through 6 connected to each other by single $(-)$ or double (=) bonds.

Attached to the $\mathrm{C}$ atom at position 1 is a carboxyl group $(\mathrm{COOH})$. Attached to the $\mathrm{C}$ atom at position

2 is a hydroxyl group $(\mathrm{OH})$.

This is the SA core structure. It is highlighted in pink. All the other SA-related compounds contain this SA core plus one or more "add ons."

For example, acetyl salicylic acid or aspirin has an acetyl group ( $\mathrm{CH} 3 \mathrm{CO})$ added on to the hydroxyl group at position 2. The presence of these different "add ons" affects how tightly different proteins bind to them and, therefore, how well or effectively they can change the activity of the bound protein. For example, the "add ons" at position 3 in amorfrutin B1 and in acetyl 3-aminoethyl SA enable our two newly identified SA targets in humans-glyceraldehyde 3-phosphate dehydrogenase and high mobility group box 1-to binding much more tightly to these SA-related compounds than to SA. pain, fever, and inflammation. It lowers the risk of heart attack, stroke, and certain cancers.

After more than a century of human use, researchers are still discovering how aspirin affects the body.

For thousands of years, people in many different cultures used plants containing aspirin-like compounds. For example, about 2500 years ago the Greek physician Hippocrates prescribed willow bark to treat fever and pain. For centuries in Europe, people grew meadowsweet to treat pain and inflammation. Willow and meadowsweet contain high levels of aspirin-like compounds called salicin and methyl salicylate, respectively. Aspirin, salicin, and methyl salicylate are all rapidly converted into a substance called salicylic acid (SA for short) in the human body.

By the 1800s, scientists knew that SA was the component derived from plants that relieved pain and fever. However, its long-term use at high doses caused stomach problems in some people. In 1897, a chemist at a company called Bayer added a chemical modification called an acetyl group $\left(\mathrm{CH}_{3} \mathrm{CO}\right)$ to SA, turning it into acetyl salicylic acid. Bayer called this new substance aspirin. Aspirin causes fewer stomach problems. The chemical structure of aspirin, SA, and other similar substances are shown in Figure 1.

\section{WHAT IS SA AND WHAT ARE ITS FUNCTIONS IN PLANTS?}

All plants produce SA. SA functions as an important hormone in plants. Hormones are compounds that control biological processes. Some of the many processes that SA affects in plants are shown in Figure 2. Importantly, SA controls the plant's immune responses to infection.

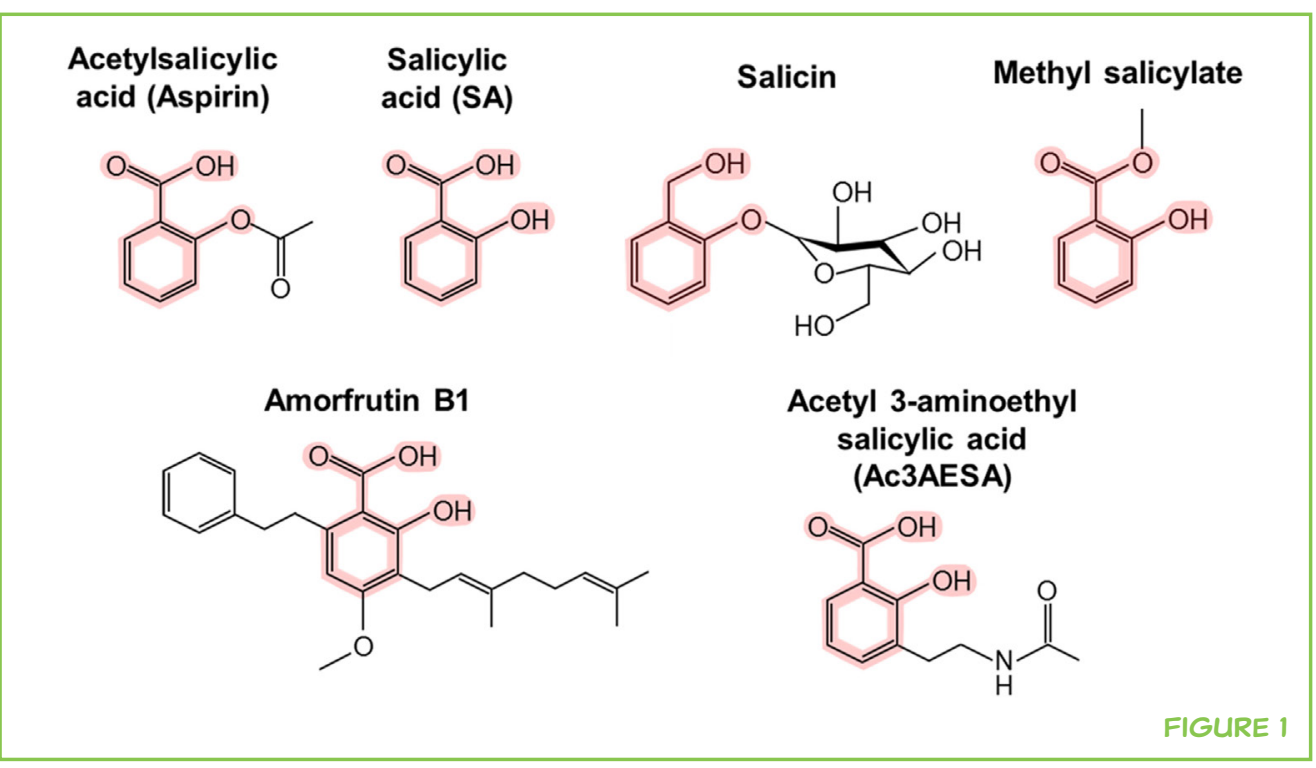


FIGURE 2

Some of the roles of salicylic acid in plants.

\section{HORMONES}

Are substances produced in an organism and transported in tissue fluids, such as blood in animal or sap in plants, to stimulate specific cells or tissues into action.

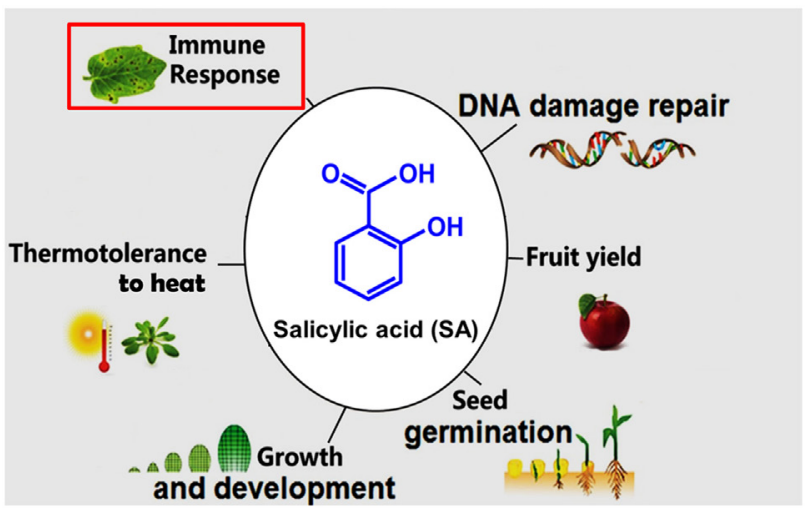

FIGURE 2

Most hormones affect biological processes in plants and animals by binding to one or a small number of proteins, called receptors. Surprisingly, SA appears to act differently. We used new laboratory methods to sift through most of the 20,000 different proteins in a plant cell. We discovered dozens of proteins that bind SA. Binding to SA alters the activity of these proteins [1]. We refer to the proteins that bind to SA as SA-binding proteins (SABPs) or SA targets.

The strength or tightness of the SABP's binding to SA is called affinity. Some SABPs have high affinity for SA, that is, they bind SA tightly. This means that even when levels of SA are low, these SABPs will be bound to SA, which changes their activities. Other SABPs have low affinity. As a result, they bind SA and change their activity only when SA levels are high. Importantly, SA levels in plants can vary greatly. SA levels in plants can be different from one location to another within one cell, they can vary in different plant tissues, at different developmental stages of the plant, or when the plant is responding to different environmental cues, such as infection. This means that the activities of the various SABPs will be affected differently, depending on where they are located, the developmental stage of the plant, and the external/environmental conditions. This combination of SABPs with a wide range of affinities for SA and the varying SA levels within the plant means that there are many different ways that SA can affect plants.

\section{WHY DOES SA AFFECT HUMANS?}

So why does a plant hormone have so many effects on humans? The majority of animals, including humans, eat plants. This exposes them to SA and related compounds on a regular basis. In addition, some studies suggest that animals produce their own SA from compounds present in high amounts in certain foods. The continued presence of SA in the bodies of animals, resulting from both diet and possibly their own production of SA, might have led, over time, to the evolution of multiple SA targets in animals. If future studies confirm this hypothesis, then even more SA targets present in both plants and animals will be identified. Learning more about these targets will help scientists determine 


\section{CYCLOOXY-}

\section{GENASES}

Are proteins which make prostaglandins.

\section{PROSTAGLANDINS}

Are hormone-like substances that participate in a wide range of body functions, including modulation of inflammation.

\section{FIGURE 3}

Why aspirin must have targets in addition to the cyclooxygenases. the mechanisms through which SA functions. This knowledge should provide clues for creating highly successful strategies to control disease in plants and animals.

\section{HOW DOES ASPIRIN WORK IN HUMANS?}

In the 1970s, scientists thought that they had figured out how aspirin works. What they discovered was only a small part of what SA does and how it does it. In the early 1970s, English scientist John Vane and his coworkers discovered that aspirin stops the activity of certain proteins called cyclooxygenases. Cyclooxygenases make substances called prostaglandins. Prostaglandins are hormone-like compounds that can cause pain, fever, and inflammation. This important discovery won Vane the Nobel Prize in 1982. Since this discovery, most scientists and physicians have believed that aspirin works by stopping the activity of cyclooxygenases.

The idea that aspirin's only function in human/animals is to block cyclooxygenases ignores four important facts (Figure 3). First and most importantly, even though SA is not very good at inhibiting the cyclooxygenases, aspirin and SA have nearly the same effects on the symptoms of humans/animals who receive it. Second, in humans, aspirin is converted within minutes into SA. SA is stable for many hours. Third, long before there was aspirin, SA was the major drug used to reduce pain, fever, and inflammation. Fourth, even before there was man-made SA, plants containing high levels of SA and related compounds were used for thousands of years by many different cultures to reduce pain, fever, and inflammation. They are still being used today. Thus, there must be other targets, in addition to the cyclooxygenases, through which SA and aspirin express their many positive effects.

\section{WHAT ELSE DOES ASPIRIN OR SA DO?}

To find the additional targets of SA/aspirin in humans, we used the same methods that we previously used to search for SA targets in plants. We discovered several new targets. Importantly, these targets are associated with some of the

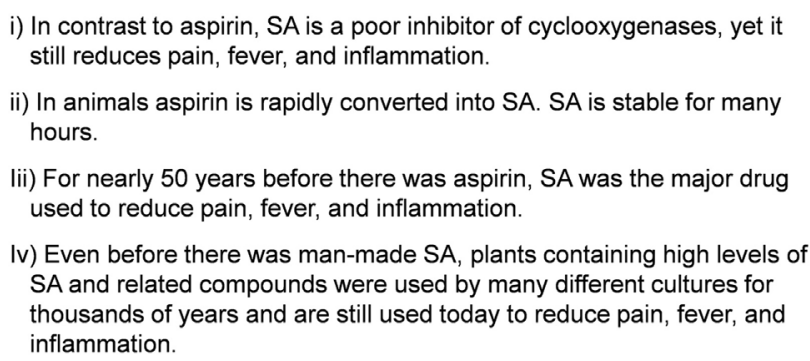

ii) In animals aspirin is rapidly converted into SA. SA is stable for many hours.

lii) For nearly 50 years before there was aspirin, SA was the major drug used to reduce pain, fever, and inflammation.

Iv) Even before there was man-made SA, plants containing high levels of $S A$ and related compounds were used by many different cultures for thousands of years and are still used today to reduce pain, fever, and inflammation.

Thus, there must be other targets, in addition to the cyclooxygenases, through which SA/aspirin works! 
most common and devastating human diseases. These targets include glyceraldehyde 3-phosphate dehydrogenase (GAPDH), a very important protein needed for cells to produce energy [2]. Some plant and animal viruses, including viruses that cause stunting (slowed growth) in tomato plants and those that cause a liver disease called hepatitis in humans, use GAPDH to reproduce inside the host cell. In our experiments, we showed that plant GAPDH binds to $\mathrm{SA}$, and this binding blocks the growth of a virus called tomato bushy stunt virus [3]. We believe that SA will also suppress reproduction of hepatitis virus. Also, GAPDH is a major suspect in several brain diseases. These brain diseases include Huntington's, Parkinson's, and Alzheimer's diseases. We found that human GAPDH also binds to SA, and this binding helps to prevent the brain cells from dying [2].

Our search for SA targets also uncovered another protein, called high mobility group box 1 (HMGB1). This protein exists in high levels in the nucleus of cells, where the DNA (genetic material) is located. HMGB1 helps pack DNA into the nucleus. When HMGB1 leaks out of cells because tissues are damaged, it activates the immune system in animals. Specifically, HMGB1 recruits immune cells to the damaged tissue. HMGB1 also stimulates the immune cells to produce proteins which stimulate inflammation. The resulting inflammation protects the damaged tissue against infection. However, sometimes the body does not properly control inflammation. This can lead to the development of many inflammation-associated diseases. These include heart disease, arthritis, inflammatory bowel disorders, and certain cancers. We discovered that HMGB1 binds SA, and this binding blocks the pro-inflammatory activities of HMGB1 [4].

All cells with a nucleus, including plant cells, have HMGB1-related proteins. We discovered that plant HMGB3 also activates immune responses in plants when it is released from cells. Like human HMGB1, HMGB3 binds SA. This binding blocks the ability of HMGB3 to activate the plant's immune responses [5]. Interestingly, both HMGBs and GAPDH have similar activities in plants and humans. These activities are suppressed by SA.

\section{HOPE FOR A BETTER ASPIRIN!}

Our studies with human HMBG1 and GAPDH led to the discovery of new man-made and natural compounds created from SA. HMBG1 and GAPDH bind to these new SA-related compounds tighter than they bind to SA. As a result, these new SA-related compounds are 10-100 times more potent than SA in altering the activities of HMBG1 and GAPDH. The natural SA-related compounds are called amorfrutins. Amorfrutins come from the Chinese medicinal plant Glycyrrhiza foetida. This plant is commonly called licorice. A man-made compound called acetyl 3-aminoethyl SA was designed based on the structure of the amorfrutins. Both amorfrutins and acetyl 3-aminoethyl SA contain similar chemical "add ons" attached to them on C atom at 
position 3 (Figure 1). HMGB1 and GAPDH bind to these new compounds tighter than they bind to SA because of these add ons. As a result, these new SA-related compounds are more potent inhibitors of HMGB1 and GAPDH. This discovery shows that the development of SA-based drugs that work better than SA itself is possible.

\section{ACKNOWLEDGMENT}

The authors thank Dr. Hyong Woo Choi for assistance in preparing the figures. The work summarized above that was carried out by the author and his coworkers was funded by the US National Science Foundation grant numbers MCB-9310371, MCB-9904660, IBN-0110272, IBN-0241531, DBI0500550, IOS-052360, and IOS-0820405.

\section{ORIGINAL SOURCE ARTICLE}

Klessig, D. F., Tian, M., and Choi, H. W. 2016. Multiple targets of salicylic acid and its derivatives in plants and animals. Front. Immunol. 7:1-10. doi:10.3389/ fimmu.2016.00206

\section{REFERENCES}

1. Manohar, M., Tian, M., Moreau, M., Park, S. W., Choi, H. W., Fei, Z., et al. 2015. Identification of multiple salicylic acid-binding proteins using two high throughput screens. Front. Plant Sci. 5:777. doi:10.3389/fpls.2014.00777

2. Choi, H. W., Tian, M., Manohar, M., Harraz, M. M., Park, S. W., Schroeder, F. C., et al. 2015. Human GAPDH is a target of aspirin's primary metabolite salicylic acid and its derivatives. PLOS ONE. doi:10.1371/journal.pone.0143447

3. Tian, M., Sasvari, Z., Gonzalez, P. A., Friso, G., Rowland, E., Liu, X., et al. 2015. Salicylic acid inhibits the replication of tomato bushy stunt virus by directly targeting a host component in the replication complex. Mol. Plant Microbe Interact. 28:37986. doi:10.1094/MPMI-09-14-0259-R

4. Choi, H. W., Tian, M., Song, F., Venereau, E., Preti, A., Park, S. W., et al. 2015. Aspirin's active metabolite salicylic acid targets human high mobility group box 1 to modulate inflammatory responses. Mol. Med. 21:526-35. doi:10.2119/ molmed.2015.00148

5. Choi, H. W., Manohar, M., Manosalva, P., Tian, M., Moreau, M., and Klessig, D. F. 2016. Activation of plant innate immunity by extracellular high mobility group box 3 and its inhibition by salicylic acid. PLoS Pathog. 12:e1005518. doi:10.1371/journal. ppat.1005518

SUBMITTED: 08 February 2017; ACCEPTED: 12 April 2017;

PUBLISHED ONLINE: 17 May 2017.

EDITED BY: Pasquale Maffia, University of Glasgow, UK 


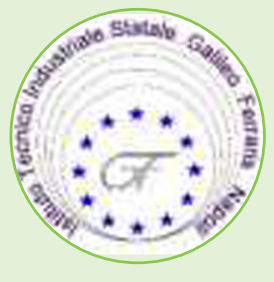

CITATION: Klessig DF (2017) How Does Aspirin Work in Plants and Humans? Front. Young Minds 5:14. doi:10.3389/frym.2017.00014

CONFLICT OF INTEREST STATEMENT: The author declares that the research was conducted in the absence of any commercial or financial relationships that could be construed as a potential conflict of interest.

COPYRIGHT (C) 2017 Klessig. This is an open-access article distributed under the terms of the Creative Commons Attribution License (CC BY). The use, distribution or reproduction in other forums is permitted, provided the original author(s) or licensor are credited and that the original publication in this journal is cited, in accordance with accepted academic practice. No use, distribution or reproduction is permitted which does not comply with these terms.

\section{REVIEWED BY}

\section{ITI GALILEO FERRARIS, 14-15 YEARS OLD}

We are a second class of a High School in Naples, Italy. Our school is deeply oriented towards technical and scientific subjects for which English obviously plays a key role.

\section{AUTHOR}

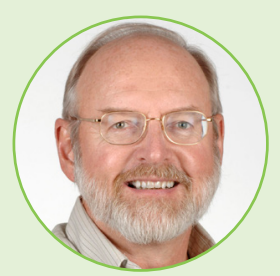

\section{DAN KLESSIG}

Dan Klessig trained under Nobel Laureate James Watson at Harvard. His early research on human adenoviruses, which provided some of the first evidence for split genes and RNA splicing, began a remarkable career of discovery and innovation. In the early 1980s, Klessig expanded his research to plant pathology. His research, which spans 40 years and 240 publications on human and plant diseases, provides a unique perspective, which has enabled him to bridge the wide gap between these two fields and gain unusual insights into the common biology and biochemistry humans share with plants. *dfk8@cornell.edu 